\title{
Low-energy constants from charmed baryons on QCD lattices
}

\author{
Yonggoo Heo, ${ }^{1}$ Xiao-Yu Guo, ${ }^{2}$ and Matthias F. M. Lutz ${ }^{2,3}$ \\ ${ }^{1}$ Suranaree University of Technology, Nakhon Ratchasima, 30000, Thailand \\ ${ }^{2}$ GSI Helmholtzzentrum für Schwerionenforschung GmbH, Planckstraße 1, 64291 Darmstadt, Germany \\ ${ }^{3}$ Technische Universität Darmstadt, D-64289 Darmstadt, Germany
}

(Received 11 October 2019; accepted 21 February 2020; published 17 March 2020)

\begin{abstract}
We study the light quark-mass dependence of charmed baryon masses as measured by various QCD lattice collaborations. A global fit to such data based on the chiral SU(3) Lagrangian is reported on. All lowenergy constants that are relevant at next-to-next-to-next-to-leading order are determined from the lattice datasets where constraints from sum rules as they follow from large- $N_{c}$ QCD at subleading order are considered. The expected hierarchy for the low-energy constants in the $1 / N_{c}$ expansion is confirmed by our global fits to the lattice data. With our results, the low-energy interaction of the Goldstone bosons with the charmed baryon ground states is well constrained and the path toward realistic coupled-channel computations in this sector of QCD is prepared.
\end{abstract}

DOI: 10.1103/PhysRevD.101.054506

\section{INTRODUCTION}

The challenge of modern particle physics is how to connect the wealth of experimental data on hadron spectroscopy to the fundamental theory of strong interactions. While lattice QCD approaches made significant advances in the last decade, it is still a grand endeavor to compute the hadronic excitation spectrum on QCD lattices directly. On the one hand, the reliable determination of finite-box energy levels requires the consideration of all open hadronic channels as it was demonstrated repeatedly that the omission of some channels may distort the finite-box spectrum significantly [1]. On the other hand, the translation of such finite-box spectra to the physics in the laboratory requires extensive coupled-channel studies that resemble to some extent the partial-wave analyses required for the experimental datasets.

Given the complexity of this problem, it may be of advantage to systematically combine the strength of lattice QCD approaches with that of effective field theory (EFT). While an EFT is quite efficient to work in symmetry aspects of QCD and gets close to data taken in the laboratory, it is a priori ignorant about various dynamical aspects of QCD. This is reflected in the large number of low-energy constants that arise at subleading orders. However, we argue that this main drawback can be overcome by the use of existing lattice datasets. Since the simulations are

Published by the American Physical Society under the terms of the Creative Commons Attribution 4.0 International license. Further distribution of this work must maintain attribution to the author(s) and the published article's title, journal citation, and DOI. Funded by SCOAP ${ }^{3}$. performed at various unphysical quark masses, information is generated that is instrumental in determining large sets of low-energy constants.

Such programs have already been successfully set up for masses of baryons and mesons in their ground states with $J^{P}=\frac{1}{2}+, \frac{3}{2}{ }^{+}$and $J^{P}=0^{-}, 1^{-}$quantum numbers [2-5]. Significant sets of low-energy parameters to be used in flavor SU(3) chiral Lagrangians were established from the available lattice data on such hadron masses. So far, results are available for mesons and baryons composed of up, down, and strange quarks $[2,5,6]$. In addition, a complete set of relevant low-energy constants in the open-charm sector of QCD was established in [3].

The purpose of this work is to present results for the lowenergy constants of the chiral Lagrangian formulated for charmed baryons [7-10]. Like in the open-charm meson sector, the coupled-channel dynamics of the leading order chiral Lagrangian predicts the existence of various dynamically generated states $[9,11,12]$. Such states carry $J^{P}=\frac{1}{2}^{+}$ and $J^{P}=\frac{3}{2}{ }^{+}$quantum numbers and may be formed in flavor exotic multiplets. Thus, it is an important challenge to estimate the size of chiral correction terms from QCD studies. At next-to-next-to-next-to-leading order $\left(\mathrm{N}^{3} \mathrm{LO}\right)$, we count 54 parameters that need to be determined in computations of the charmed baryon masses. Given the limited dataset that is provided so far [13-19], it is important to derive additional constraints from QCD that further constrain a fit of the low-energy constants to such data. A corresponding framework was worked out already in $[4,20]$. From a systematic consideration of sum rules that arise in the $1 / N_{c}$ expansion of QCD at subleading order, the number of independent low-energy constants was reduced significantly down to 23 parameters only [20]. 


\section{CHIRAL LAGRANGIAN WITH CHARMED BARYON FIELDS}

The chiral Lagrangian combined with appropriate counting rules leads to systematic results in low-energy hadron physics. In the following, we recall the leading order (LO) terms in the open-charm baryon sector of QCD $[7,8,10]$. It is convenient to decompose the fields into their isospin multiplets with

$$
\begin{aligned}
\Phi & =\tau \cdot \pi(140)+\alpha^{\dagger} \cdot K(494)+K^{\dagger}(494) \cdot \alpha+\eta(547) \lambda_{8}, \\
\sqrt{2} B_{[\overline{[}]} & =\frac{1}{\sqrt{2}} \alpha^{\dagger} \cdot \Xi_{c}(2470)-\frac{1}{\sqrt{2}} \Xi_{c}^{T}(2470) \cdot \alpha+i \tau_{2} \Lambda_{c}(2284), \\
\sqrt{2} B_{[6]} & =\frac{1}{\sqrt{2}} \alpha^{\dagger} \cdot \Xi_{c}^{\prime}(2580)+\frac{1}{\sqrt{2}} \Xi_{c}^{\prime T}(2580) \cdot \alpha+\Sigma_{c}(2455) \cdot \tau i \tau_{2}+\frac{\sqrt{2}}{3}\left(1-\sqrt{3} \lambda_{8}\right) \Omega_{c}(2704), \\
\sqrt{2} B_{[6]}^{\mu} & =\frac{1}{\sqrt{2}} \alpha^{\dagger} \cdot \Xi_{c}^{\mu}(2645)+\frac{1}{\sqrt{2}} \Xi_{c}^{T, \mu}(2645) \cdot \alpha+\Sigma_{c}^{\mu}(2520) \cdot \tau i \tau_{2}+\frac{\sqrt{2}}{3}\left(1-\sqrt{3} \lambda_{8}\right) \Omega_{c}^{\mu}(2770), \\
\alpha^{\dagger} & =\frac{1}{\sqrt{2}}\left(\lambda_{4}+i \lambda_{5}, \lambda_{6}+i \lambda_{7}\right), \quad \tau=\left(\lambda_{1}, \lambda_{2}, \lambda_{3}\right),
\end{aligned}
$$

where the matrices $\lambda_{i}$ are the standard Gell-Mann generators of the SU(3) algebra. The numbers in the brackets recall the approximate masses of the particles in units of $\mathrm{MeV}$. There are the kinetic terms,

$$
\begin{aligned}
\mathcal{L}^{(1)}= & \operatorname{tr} \bar{B}_{[6]}\left(\gamma^{\mu} i D_{\mu}-M_{[6]}^{(1 / 2)}\right) B_{[6]}-\operatorname{tr}\left(\bar{B}_{[6]}^{\mu}\left(\left[i \not D-M_{[6]}^{(3 / 2)}\right] g_{\mu \nu}-i\left(\gamma_{\mu} D_{\nu}+\gamma_{\nu} D_{\mu}\right)+\gamma_{\mu}\left[i \not D+M_{[6]}^{(3 / 2)}\right] \gamma_{\nu}\right) B_{[6]}^{\nu}\right) \\
& +\operatorname{tr} \bar{B}_{[\overline{3}]}\left(\gamma^{\mu} i D_{\mu}-M_{[\overline{3}]}^{(1 / 2)}\right) B_{[\overline{[}]}+F_{[66]} \operatorname{tr} \bar{B}_{[6]} \gamma^{\mu} \gamma_{5} i U_{\mu} B_{[6]}+F_{[\overline{3} \overline{3}]} \operatorname{tr} \bar{B}_{[\overline{3}]} \gamma^{\mu} \gamma_{5} i U_{\mu} B_{[\overline{3}]}+F_{[\overline{3} 6]} \operatorname{tr}\left(\bar{B}_{[6]} \gamma^{\mu} \gamma_{5} i U_{\mu} B_{[\overline{3}]}+\text { H.c. }\right) \\
& +C_{[66]} \operatorname{tr}\left(\bar{B}_{[6]}^{\mu} i U_{\mu} B_{[6]}+\text { H.c. }\right)+C_{[\overline{[} 6]} \operatorname{tr}\left(\bar{B}_{[6]}^{\mu} i U_{\mu} B_{[\overline{3}]}+\text { H.c. }\right)-H_{[66]} \operatorname{tr} \bar{B}_{[6]}^{\alpha} g_{\alpha \beta} \gamma^{\mu} \gamma_{5} i U_{\mu} B_{[6]}^{\beta}, \\
U_{\mu}= & \frac{1}{2} u^{\dagger}\left(\partial_{\mu} e^{i \frac{\Phi}{f}}\right) u^{\dagger}-\frac{i}{2} u^{\dagger}\left(v_{\mu}+a_{\mu}\right) u+\frac{i}{2} u\left(v_{\mu}-a_{\mu}\right) u^{\dagger}, \\
D_{\mu} B= & \partial_{\mu} B+\Gamma_{\mu} B+B \Gamma_{\mu}^{\dagger}, \quad u=e^{i \frac{\Phi}{2 f}}, \\
\Gamma_{\mu}= & \frac{1}{2} u^{\dagger}\left[\partial_{\mu}-i\left(v_{\mu}+a_{\mu}\right)\right] u+\frac{1}{2} u\left[\partial_{\mu}-i\left(v_{\mu}-a_{\mu}\right)\right] u^{\dagger},
\end{aligned}
$$

and six structures which parametrize the three-point interactions of the Goldstone bosons with the charmed baryon fields [7,8]. At leading order in a chiral expansion, the bare masses $M_{[6]}^{(1 / 2)}, M_{[6]}^{(3 / 2)}$, and $M_{[\overline{3}]}^{(1 / 2)}$ may be identified with the flavor average of the sextet and antitriplet baryon masses.

At next-to-leading order (NLO), there are symmetry conserving and symmetry breaking terms [10,20]. A complete list of chiral symmetry conserving $Q^{2}$ counter terms was given in $[10,20]$. In these works, the $Q^{2}$ counter terms are grouped according to their Dirac structure. Here, we display the scalar and vector terms relevant for our study only,

$$
\begin{aligned}
\mathcal{L}^{(S)}= & -g_{0, \overline{3} \overline{3}]}^{(S)} \operatorname{tr}\left(\bar{B}_{[\overline{3}]} B_{[\overline{3}]}\right) \operatorname{tr}\left(U_{\mu} U^{\mu}\right)-g_{D, \overline{3} \overline{3}]}^{(S)} \operatorname{tr}\left(\bar{B}_{[\overline{3}]}\left\{U_{\mu}, U^{\mu}\right\} B_{[\overline{3}]}\right)-g_{0,[66]}^{(S)} \operatorname{tr}\left(\bar{B}_{[6]} B_{[6]}\right) \operatorname{tr}\left(U_{\mu} U^{\mu}\right)-g_{1,[66]}^{(S)} \operatorname{tr}\left(\bar{B}_{[6]} U^{\mu} B_{[6]} U_{\mu}^{T}\right) \\
& -g_{D,[66]}^{(S)} \operatorname{tr}\left(\bar{B}_{[6]}\left\{U_{\mu}, U^{\mu}\right\} B_{[6]}\right)-g_{D,[\overline{3} 6]}^{(S)} \operatorname{tr}\left(\bar{B}_{[6]}\left\{U_{\mu}, U^{\mu}\right\} B_{[\overline{3}]}+\text { H.c. }\right)+h_{0,[66]}^{(S)} \operatorname{tr}\left(\bar{B}_{[6]}^{\mu} g_{\mu \nu} B_{[6]}^{\nu}\right) \operatorname{tr}\left(U_{\alpha} U^{\alpha}\right) \\
& +h_{1,[66]}^{(S)} \operatorname{tr}\left(\bar{B}_{[6]}^{\mu} B_{[6]}^{\nu}\right) \operatorname{tr}\left(U_{\mu} U_{\nu}\right)+h_{2,[66]}^{(S)} \operatorname{tr}\left(\bar{B}_{[6]}^{\mu} g_{\mu \nu}\left\{U^{\alpha}, U_{\alpha}\right\} B_{[6]}^{\nu}\right)+h_{3,[66]}^{(S)} \operatorname{tr}\left(\bar{B}_{[6]}^{\mu}\left\{U_{\mu}, U_{\nu}\right\} B_{[6]}^{\nu}\right) \\
& +h_{4,[66]}^{(S)} \operatorname{tr}\left(\bar{B}_{[6]}^{\mu} g_{\mu \nu} U^{\alpha} B_{[6]}^{\nu} U_{\alpha}^{T}\right)+\frac{1}{2} h_{5,[66]}^{(S)} \operatorname{tr}\left(\bar{B}_{[6]}^{\mu} U_{\nu} B_{[6]}^{\nu} U_{\mu}^{T}+\bar{B}_{[6]}^{\mu} U_{\mu} B_{[6]}^{\nu} U_{\nu}^{T}\right),
\end{aligned}
$$




$$
\begin{aligned}
\mathcal{L}^{(V)}= & -\frac{1}{2} g_{0,[\overline{3} \overline{3}]}^{(V)} \operatorname{tr}\left(\bar{B}_{[\overline{3}]} i \gamma^{\alpha}\left(D^{\beta} B_{[\overline{3}]}\right) \operatorname{tr}\left(U_{\beta} U_{\alpha}\right)\right)-\frac{1}{2} g_{1,[\overline{3} \overline{3}]}^{(V)} \operatorname{tr}\left(\bar{B}_{[\overline{3}]} i \gamma^{\alpha} U_{\beta}\left(D^{\beta} B_{[\overline{3}]}\right) U_{\alpha}^{T}+\bar{B}_{[\overline{3}]} i \gamma^{\alpha} U_{\alpha}\left(D^{\beta} B_{[\overline{3}]}\right) U_{\beta}^{T}\right) \\
& -\frac{1}{2} g_{D,[\overline{3} \overline{3}]}^{(V)} \operatorname{tr}\left(\bar{B}_{[\overline{3}]} i \gamma^{\alpha}\left\{U_{\alpha}, U_{\beta}\right\}\left(D^{\beta} B_{[\overline{3}]}\right)\right)-\frac{1}{2} g_{D,[\overline{3} 6]}^{(V)} \operatorname{tr}\left(\bar{B}_{[6]} i \gamma^{\alpha}\left\{U_{\alpha}, U_{\beta}\right\}\left(D^{\beta} B_{[\overline{3}]}\right)-\left(D^{\beta} \bar{B}_{[6]}\right) i \gamma^{\alpha}\left\{U_{\alpha}, U_{\beta}\right\} B_{[\overline{3}]}\right) \\
& -\frac{1}{2} g_{0,[66]}^{(V)}\left(\operatorname{tr}\left(\bar{B}_{[6]} i \gamma^{\alpha}\left(D^{\beta} B_{[6]}\right)\right) \operatorname{tr}\left(U_{\beta} U_{\alpha}\right)\right)-\frac{1}{4} g_{1,[66]}^{(V)} \operatorname{tr}\left(\bar{B}_{[6]} i \gamma^{\alpha} U_{\beta}\left(D^{\beta} B_{[6]}\right) U_{\alpha}^{T}+\bar{B}_{[6]} i \gamma^{\alpha} U_{\alpha}\left(D^{\beta} B_{[6]}\right) U_{\beta}^{T}\right) \\
& -\frac{1}{2} g_{D,[66]}^{(V)} \operatorname{tr}\left(\bar{B}_{[6]} i \gamma^{\alpha}\left\{U_{\alpha}, U_{\beta}\right\}\left(D^{\beta} B_{[6]}\right)\right)+\frac{1}{2} h_{0,[66]}^{(V)} \operatorname{tr}\left(\bar{B}_{[6]}^{\mu} g_{\mu \nu} i \gamma^{\alpha}\left(D^{\beta} B_{[6]}^{\nu}\right) \operatorname{tr}\left(U_{\alpha} U_{\beta}\right)\right) \\
& +\frac{1}{4} h_{1,[66]}^{(V)} \operatorname{tr}\left(\bar{B}_{[6]}^{\mu} g_{\mu \nu} i \gamma^{\alpha} U_{\beta}\left(D^{\beta} B_{[6]}^{\nu}\right) U_{\alpha}^{T}+\bar{B}_{[6]}^{\mu} g_{\mu \nu} i \gamma^{\alpha} U_{\alpha}\left(D^{\beta} B_{[6]}^{\nu}\right) U_{\beta}^{T}\right)+\frac{1}{2} h_{2,[66]}^{(V)} \operatorname{tr}\left(\bar{B}_{[6]}^{\mu} g_{\mu \nu} i \gamma^{\alpha}\left\{U_{\alpha}, U_{\beta}\right\}\left(D^{\beta} B_{[6]}^{\nu}\right)\right)+\text { H.c., }
\end{aligned}
$$

where further possible terms that are redundant owing to the on-shell conditions of spin- $\frac{3}{2}$ fields with $\gamma_{\mu} B_{[6]}^{\mu}=0$ and $\partial_{\mu} B_{[6]}^{\mu}=0$ are eliminated systematically. We note that the terms in (3) imply contributions to the charmed baryon masses that do depend on the choice of the renormalization scale. Such terms need to be balanced by a set of symmetry breaking counter terms that render the charmed baryon masses renormalization scale independent.

We turn to the terms that break chiral symmetry explicitly. There are seven symmetry breaking counter terms at order $Q^{2}$ and 16 terms of order $Q^{4}$. We recall from $[10,20]$

$$
\begin{aligned}
\mathcal{L}_{\chi}^{(2)}= & b_{1,[\overline{3} \overline{3}]} \operatorname{tr}\left(\bar{B}_{[\overline{3}]} B_{[\overline{3}]}\right) \operatorname{tr}\left(\chi_{+}\right)+b_{2,[\overline{3} \overline{3}]} \operatorname{tr}\left(\bar{B}_{[\overline{3}]} \chi_{+} B_{[\overline{3}]}\right)+b_{1,[\overline{3} 6]} \operatorname{tr}\left(\bar{B}_{[6]} \chi_{+} B_{[\overline{[}]}+\text { H.c. }\right)+b_{1,[66]} \operatorname{tr}\left(\bar{B}_{[6]} B_{[6]}\right) \operatorname{tr}\left(\chi_{+}\right) \\
& +b_{2,[66]} \operatorname{tr}\left(\bar{B}_{[6]} \chi_{+} B_{[6]}\right)-d_{1,[66]} \operatorname{tr}\left(g_{\mu \nu} \bar{B}_{[6]}^{\mu} B_{[6]}^{\nu}\right) \operatorname{tr}\left(\chi_{+}\right)-d_{2,[66]} \operatorname{tr}\left(g_{\mu \nu} \bar{B}_{[6]}^{\mu} \chi_{+} B_{[6]}^{\nu}\right), \\
\mathcal{L}_{\chi}^{(4)}= & c_{1,[\overline{3} \overline{3}]} \operatorname{tr}\left(\bar{B}_{[\overline{[}]} B_{[\overline{3}]}\right) \operatorname{tr}\left(\chi_{+}^{2}\right)+c_{2,[\overline{3} \overline{3}]} \operatorname{tr}\left(\bar{B}_{[\overline{3}]} B_{[\overline{3}]}\right)\left(\operatorname{tr} \chi_{+}\right)^{2}+c_{3,[\overline{3} \overline{3}]} \operatorname{tr}\left(\bar{B}_{[\overline{[}]} \chi_{+} B_{[\overline{3}]}\right) \operatorname{tr}\left(\chi_{+}\right)+c_{4,[\overline{3} \overline{3}]} \operatorname{tr}\left(\bar{B}_{[\overline{3}]} \chi_{+}^{2} B_{[\overline{3}]}\right) \\
& +c_{1,[66]} \operatorname{tr}\left(\bar{B}_{[6]} B_{[6]}\right) \operatorname{tr}\left(\chi_{+}^{2}\right)+c_{2,[66]} \operatorname{tr}\left(\bar{B}_{[6]} B_{[6]}\right)\left(\operatorname{tr} \chi_{+}\right)^{2}+c_{3,[66]} \operatorname{tr}\left(\bar{B}_{[6]} \chi_{+} B_{[6]}\right) \operatorname{tr}\left(\chi_{+}\right)+c_{4,[66]} \operatorname{tr}\left(\bar{B}_{[6]} \chi_{+}^{2} B_{[6]}\right) \\
& +c_{5,[66]} \operatorname{tr}\left(\bar{B}_{[6]} \chi_{+} B_{[6]} \chi_{+}^{T}\right)+c_{1,[\overline{3} 6]} \operatorname{tr}\left(\bar{B}_{[6]} \chi_{+} B_{[\overline{3}]}+\text { H.c. }\right) \operatorname{tr}\left(\chi_{+}\right)+c_{2,[\overline{3} 6]} \operatorname{tr}\left(\bar{B}_{[6]} \chi_{+}^{2} B_{[\overline{3}]}+\text { H.c. }\right) \\
& -e_{1,[66]} \operatorname{tr}\left(\bar{B}_{[6]}^{\mu} g_{\mu \nu} B_{[6]}^{\nu}\right) \operatorname{tr}\left(\chi_{+}^{2}\right)-e_{2,[66]} \operatorname{tr}\left(\bar{B}_{[6]}^{\mu} g_{\mu \nu} B_{[6]}^{\nu}\right)\left(\operatorname{tr} \chi_{+}\right)^{2}-e_{3,[66]} \operatorname{tr}\left(\bar{B}_{[6]}^{\mu} g_{\mu \nu} \chi_{+} B_{[6]}^{\nu}\right) \operatorname{tr}\left(\chi_{+}\right)-e_{4,[66]} \operatorname{tr}\left(\bar{B}_{[6]}^{\mu} g_{\mu \nu} \chi_{+}^{2} B_{[6]}^{\nu}\right) \\
& -e_{5,[66]} \operatorname{tr}\left(\bar{B}_{[6]}^{\mu} g_{\mu \nu} \chi_{+} B_{[6]}^{\nu} \chi_{+}^{T}\right) . \\
\chi_{+}= & \frac{1}{2}\left(u \chi_{0} u+u^{\dagger} \chi_{0} u^{\dagger}\right),
\end{aligned}
$$

with $\quad \chi_{0}=2 B_{0} \operatorname{diag}\left(m, m, m_{s}\right) \quad$ proportional to the quark-mass matrix. We do not consider isospin violating effects in this work. With $\mathcal{L}_{\chi}^{(4)}$, the 16 symmetry breaking counter terms that contribute to the charm baryon masses at $\mathrm{N}^{3} \mathrm{LO}$ are shown.

Altogether, we count 54 low-energy constants in this section that have to be determined by the datasets. Clearly, any additional constraints from heavy-quark spinsymmetry or large- $N_{c}$ QCD are desperately needed to arrive at any significant result. Such constraints were derived in $[10,20,21]$ to subleading order in the $1 / N_{c}$ expansion and are summarized in Appendix A of [4]. At subleading order, there remain 23 independent low-energy constants only.

\section{CHARMED BARYON MASSES FROM QCD LATTICE SIMULATIONS}

We recall our strategy how to make use of the available QCD lattice datasets on hadron ground-state masses $[2,3,5,22]$. Altogether, we consider 210 data points on lattice QCD ensembles with pion and kaon masses smaller than $600 \mathrm{MeV}$. On each ensemble, the baryon masses are provided in units of the corresponding lattice scale together with an estimate of their statistical error only. To actually perform the fits is a computational challenge. For any set of the low-energy parameters, ten coupled nonlinear equations are to be solved on each lattice ensemble considered (see [4]). We apply the evolutionary algorithm of GENEVA 1.9.0-GSI [23] with runs of a population size 2500 on 500 parallel CPU cores.

A subset of low-energy constants (LEC) is fixed by the requirement that the isospin averaged charmed baryon masses are reproduced as provided by the Particle Data Group [24]. This amounts to a nonstandard scale setting for the various lattice ensembles considered. In our work, we assume that the lattice datasets are consistent with the physical values of the charmed baryon masses. The purpose of our analysis is to extract the low-energy constants, rather than to estimate how well a dataset can be extrapolated down to the physical point. For any given $\beta$ value 
characterizing a lattice ensemble, we determine its associated lattice scale separately. From any such ensemble, we take its published pion and kaon masses as given in lattice units and derive their associated quark masses. As in our previous works $[2,3]$, we do so at the one-loop level in terms of the low-energy constants of Gasser and Leutwyler. At the physical point, the products $B_{0} m, B_{0} m_{s}$, and $L_{8}+$ $3 L_{7}$ are set by the request to reproduce the empirical pion, kaon, and eta masses. That leaves two particular combinations $2 L_{6}-L_{4}$ and $2 L_{8}-L_{5}$ undetermined only. As emphasized repeatedly, the lattice datasets on hadron masses are quite sensitive to the latter and permit an accurate determination of their values [2,3]. Consistent results were obtained from two independent analysis based on distinct lattice datasets on the hadron masses. This suggests the ranges $10^{3}\left(2 L_{6}-L_{4}\right)=0.04-0.16$ and $10^{3}\left(2 L_{8}-L_{5}\right)=$ $0.02-0.11$ at the renormalization scale $\mu=0.77 \mathrm{GeV}$. Such values are compatible with the ranges from the FLAG report [25].

Our analyses rest on the NLO large- $N_{c}$ sum rules of $[4,20]$. For instance, we recall

$$
\begin{aligned}
C_{[66]} & =\frac{\sqrt{3}}{2}\left(F_{[\overline{3} \overline{3}]}-F_{[66]}\right), \quad C_{[\overline{[} 6]}=\sqrt{3} F_{[\overline{3} 6]}, \\
H_{[66]} & =\frac{3}{2}\left(F_{[\overline{3} \overline{3}]}+F_{[66]}\right), \quad b_{1,[66]}=d_{1,[66]}=b_{1,[\overline{3} \overline{3}]} \\
b_{1,[\overline{3} 6]} & =\frac{1}{\sqrt{3}}\left(b_{2,[66]}-d_{2,[66]}\right) .
\end{aligned}
$$

TABLE I. Results for LEC in (2), (4) based on three fit scenarios. Further LEC are implied by the NLO large- $N_{c}$ sum rules of [20]. The low-energy constants $L_{n}$ are at the renorm-

\begin{tabular}{|c|c|c|c|}
\hline & Fit 1 & Fit 2 & Fit 3 \\
\hline$M_{[\overline{3}]}^{(1 / 2)}[\mathrm{Gev}]$ & $2.468\left({ }_{-1}^{+0}\right)$ & $2.424\left({ }_{-3}^{+0}\right)$ & $2.415\left(\begin{array}{c}+0 \\
-0\end{array}\right)$ \\
\hline$M_{[6]}^{(1 / 2)}[\mathrm{Gev}]$ & $2.515\left(\begin{array}{l}+1 \\
-1\end{array}\right)$ & $2.468\left({ }_{-4}^{+1}\right)$ & $2.388\left({ }_{-2}^{+4}\right)$ \\
\hline$M_{[6]}^{(3 / 2)}[\mathrm{Gev}]$ & $2.594\left({ }_{-1}^{+1}\right)$ & $2.549\left({ }_{-3}^{+1}\right)$ & $2.586\left(\begin{array}{l}+0 \\
-0\end{array}\right)$ \\
\hline$F_{[\overline{3} 6]}$ & 0.7530 & 0.7530 & -0.7530 \\
\hline$F_{[66]}$ & $0.6502\left(\begin{array}{l}+6 \\
-2\end{array}\right)$ & $0.6500\left(\begin{array}{c}+5 \\
-0\end{array}\right)$ & $0.6745\left({ }_{-1}^{+2}\right)$ \\
\hline$F_{[\overline{3} \overline{3}]}$ & $0.0053\left({ }_{-54}^{+45}\right)$ & $0.0099\left({ }_{-05}^{+73}\right)$ & $-0.0124\left(\begin{array}{l}+1 \\
-1\end{array}\right)$ \\
\hline$b_{1,[66]}\left[\mathrm{GeV}^{-1}\right]$ & $0.3372\left(\begin{array}{l}+101 \\
-115\end{array}\right)$ & $-0.2542\left({ }_{-145}^{+096}\right)$ & $0.1053\left({ }_{-41}^{+46}\right)$ \\
\hline$b_{2,[66]}\left[\mathrm{GeV}^{-1}\right]$ & $-0.3057\left(\begin{array}{l}+188 \\
-198\end{array}\right)$ & $-0.2048\left({ }_{-189}^{+377}\right)$ & $-0.5533\left({ }_{-79}^{+89}\right)$ \\
\hline$b_{2,[\overline{3} \overline{3}]}\left[\mathrm{GeV}^{-1}\right]$ & $-0.5048\left(\begin{array}{c}+188 \\
-198\end{array}\right)$ & $-0.4048\left({ }_{-189}^{+377}\right)$ & $-0.7513\left(\begin{array}{c}+89 \\
-79\end{array}\right)$ \\
\hline$d_{2,[66]}\left[\mathrm{GeV}^{-1}\right]$ & $-0.3330\left(\begin{array}{c}+188 \\
-198\end{array}\right)$ & $-0.2249\left({ }_{-179}^{+373}\right)$ & $-0.0624\left({ }_{-79}^{+89}\right)$ \\
\hline $10^{3}\left(2 L_{6}-L_{4}\right)$ & $0.0809\left(\begin{array}{l}+15 \\
-18\end{array}\right)$ & $0.0813\left({ }_{-16}^{+29}\right)$ & $0.0558\left({ }_{-2}^{+3}\right)$ \\
\hline $10^{3}\left(2 L_{8}-L_{5}\right)$ & $0.1098\left({ }_{-18}^{+18}\right)$ & $0.1498\left({ }_{-22}^{+02}\right)$ & $0.1500\left(\begin{array}{l}+0 \\
-2\end{array}\right)$ \\
\hline $10^{3}\left(L_{8}+3 L_{7}\right)$ & $-0.5023\left(\begin{array}{l}-19 \\
-7\end{array}\right)$ & $-0.5123\left(\begin{array}{l}+09 \\
-12\end{array}\right)$ & $-0.5002\left(\begin{array}{l}(+1 \\
-1\end{array}\right)$ \\
\hline$\underline{m_{s} / m}$ & $26.05\left(\begin{array}{l}+1 \\
-1\end{array}\right)$ & $25.82\left({ }_{-0}^{+1}\right)$ & $25.79\left({ }_{-0}^{+0}\right)$ \\
\hline
\end{tabular}
alization scale $\mu=0.77 \mathrm{GeV}$. We use $f=92.4 \mathrm{MeV}$ throughout this work.
While the magnitude $\left|F_{[\overline{3} 6]}\right|=0.753$ was estimated from empirical values of some hadronic decay widths parameters [4], its sign (in our phase convention as used in the derivation of the large- $N_{c}$ sum rules) remained undetermined. We will explore the implications of the two sign choices. From a recent lattice computation of axial charges of some charmed baryons [26], we may estimate $F_{[66]} \sim 0.7$, where in this case the computation does determine the sign of $F_{[66]}$ unambiguously. The condition $F_{[66]}>0.65$ is imposed throughout our analysis.

Since we have a residual uncertainty in our one-loop chiral extrapolation approach, we assign each baryon mass a systematical error that is added to its statistical error in quadrature in our various fits. Our chisquare function, $\chi^{2}$, assumes a universal systematical error for the charmed baryon masses. While such a universal ansatz for the systematic error would not be well justified in a chiral $\mathrm{SU}(2)$ extrapolation framework, we feel it to be quite reasonable in the flavor $\mathrm{SU}(3)$ case. Note that the number of independent chiral structures that characterize the neglected higher-order correction terms is significantly larger in the $\mathrm{SU}(3)$ than in a $\mathrm{SU}(2)$ case.

TABLE II. Results for the LEC in (3), (4) based on three fit

\begin{tabular}{|c|c|c|c|}
\hline & it 1 & 2 & 3 \\
\hline$c_{1,[66]}\left[\mathrm{GeV}^{-3}\right]$ & & $-0.2786\left(\begin{array}{l}+280 \\
-174\end{array}\right)$ & $-0.3466\left({ }_{-45}^{+66}\right)$ \\
\hline$c_{2,[66]}\left[\mathrm{GeV}^{-3}\right]$ & $0.0605\left(\begin{array}{l}+65 \\
-74\end{array}\right)$ & $0.1210\left(\begin{array}{l}+06 \\
-55\end{array}\right)$ & $-0.0179\left(\begin{array}{l}+36 \\
-31\end{array}\right)$ \\
\hline$c_{3,[66]}\left[\mathrm{GeV}^{-3}\right]$ & $-0.3833\left(\begin{array}{l}+68 \\
-60\end{array}\right)$ & $-0.3114\left({ }_{-085}^{+192}\right)$ & $0.1812\left(\begin{array}{l}+34 \\
-71\end{array}\right)$ \\
\hline$c_{4,[66]}\left[\mathrm{GeV}^{-3}\right]$ & $0.9850\left({ }_{-220}^{+150}\right)$ & $0.8346\left({ }_{-682}^{+027}\right)$ & $0.6941\left({ }_{-087}^{+134}\right)$ \\
\hline$c_{5,[66]}\left[\mathrm{GeV}^{-3}\right]$ & $-0.3653\left(\begin{array}{l}+15 \\
-20\end{array}\right)$ & $-0.3609\left({ }_{-42}^{+11}\right)$ & $-0.4535\left({ }_{-07}^{+15}\right)$ \\
\hline$c_{1,[\overline{3} \overline{3}]}[\mathrm{Ge}$ & $-0.7947\left({ }_{-101}^{+202}\right)$ & $-0.5741\left({ }_{-189}^{+484}\right)$ & $-0.9674\left({ }_{-45}^{+66}\right)$ \\
\hline$c_{2,[\overline{3} \overline{3}]}[\mathrm{G}$ & $0.1936\left({ }_{-74}^{+65}\right)$ & $0.2848\left(\begin{array}{c}+023 \\
-158\end{array}\right)$ & $0.3489\left({ }_{-31}^{+36}\right)$ \\
\hline$c_{3,[\overline{3} \overline{3}]}\left[\mathrm{GeV}^{-3}\right]$ & $-0.9100\left({ }_{-060}^{+146}\right)$ & $-0.8093\left({ }_{-217}^{+333}\right)$ & $-0.9759\left({ }_{-71}^{+34}\right)$ \\
\hline$e_{1,[66]}\left[\mathrm{GeV}^{-3}\right]$ & $-0.4842\left({ }_{-122}^{+136}\right)$ & $-0.2223\left({ }_{-174}^{+260}\right)$ & $-0.0643\left({ }_{-45}^{+66}\right)$ \\
\hline$e_{3,[66]}\left[\mathrm{GeV}^{-3}\right]$ & $-0.4048\left(\begin{array}{l}+75 \\
-71\end{array}\right)$ & $-0.3347\left({ }_{-107}^{+160}\right)$ & $-0.2180\left({ }_{-60}^{+84}\right)$ \\
\hline$e_{4,[66]}\left[\mathrm{GeV}^{-3}\right]$ & $0.9637\left({ }_{-210}^{+142}\right)$ & $0.8012\left({ }_{-633}^{+065}\right)$ & $0.0444\left(\begin{array}{l}+134 \\
-087\end{array}\right)$ \\
\hline$g_{0,[66]}^{(S)}[\mathrm{Ge}$ & 0.5 & $1.7697\left(_{-}^{+}\right.$ & $\left(\begin{array}{l}+44 \\
-28\end{array}\right)$ \\
\hline$g_{1,[66]}^{(S)}\left[\mathrm{GeV}^{-1}\right]$ & $0.2072\left(_{-}^{+}\right.$ & 0.4406( & $3.9737\left(\begin{array}{l}+567 \\
-115\end{array}\right)$ \\
\hline$g_{D,[66]}^{(V)}\left[\mathrm{GeV}^{-2}\right]$ & $0.4138\left({ }_{-1109}^{+0635}\right)$ & $1.2902\left(_{-}^{+}\right.$ & $-2.9384\left(\begin{array}{l}+268 \\
-316\end{array}\right)$ \\
\hline$g_{0,[\overline{3} \overline{3}]}^{(S)}[\mathrm{Ge}$ & $1.0740\left(\begin{array}{l}+614 \\
-377\end{array}\right)$ & $2.4883\left(_{-}^{+}\right.$ & $2.6536\left(\begin{array}{l}+033 \\
-158\end{array}\right)$ \\
\hline$g_{0,[\overline{3} \overline{3}]}^{(V)}\left[\mathrm{GeV}^{-2}\right]$ & $-1.7557\left({ }_{-388}^{+368}\right)$ & $-1.3281\left(_{+}^{+}\right.$ & $-5.2841\left(\begin{array}{c}+59 \\
-38\end{array}\right)$ \\
\hline$h_{1,[66]}^{(S)}\left[\mathrm{GeV}^{-1}\right]$ & $-0.0716\left({ }_{-100}^{+030}\right)$ & $-0.3608\left({ }_{-545}^{+234}\right)$ & -0.465 \\
\hline$h_{2,[66]}^{(S)}\left[\mathrm{GeV}^{-1}\right]$ & $1.0721\left(_{+}^{+}\right.$ & $1.0279{ }^{+}$ & $-0.0508\left(\begin{array}{l}+785 \\
-703\end{array}\right)$ \\
\hline$h_{4,[66]}^{(S)}\left[\mathrm{GeV}^{-1}\right]$ & $2.3125\left(\begin{array}{l}+093 \\
-168\end{array}\right)$ & $1.0016\left(_{+}^{+}\right.$ & $6.1835\left({ }_{-33}^{+00}\right)$ \\
\hline$h_{5,[66]}^{(S)}\left[\mathrm{GeV}^{-1}\right]$ & $-2.0747\left(\begin{array}{l}+525 \\
-297\end{array}\right)$ & $-0.2665\left(\begin{array}{l}+2305 \\
-0250\end{array}\right)$ & $-0.8207\left(\begin{array}{c}+67 \\
-58\end{array}\right)$ \\
\hline$h_{1,[66]}^{(V)}\left[\mathrm{GeV}^{-2}\right]$ & $-0.5500\left(\left(_{-552}^{+356}\right)\right.$ & $0.6440\left({ }_{-0335}^{+1568}\right)$ & $-6.7926\left({ }_{-076}^{+117}\right)$ \\
\hline$h_{2,[66]}^{(V)}\left[\mathrm{GeV}^{-2}\right]$ & $0.2754\left(\begin{array}{l}+151 \\
-261\end{array}\right)$ & $1.1689\left(_{-06}^{+12}\right.$ & $0.8839\left(\begin{array}{l}+07 \\
-41\end{array}\right)$ \\
\hline
\end{tabular}
scenarios. Further LEC are implied by the NLO large- $N_{c}$ sum rules of [20]. 


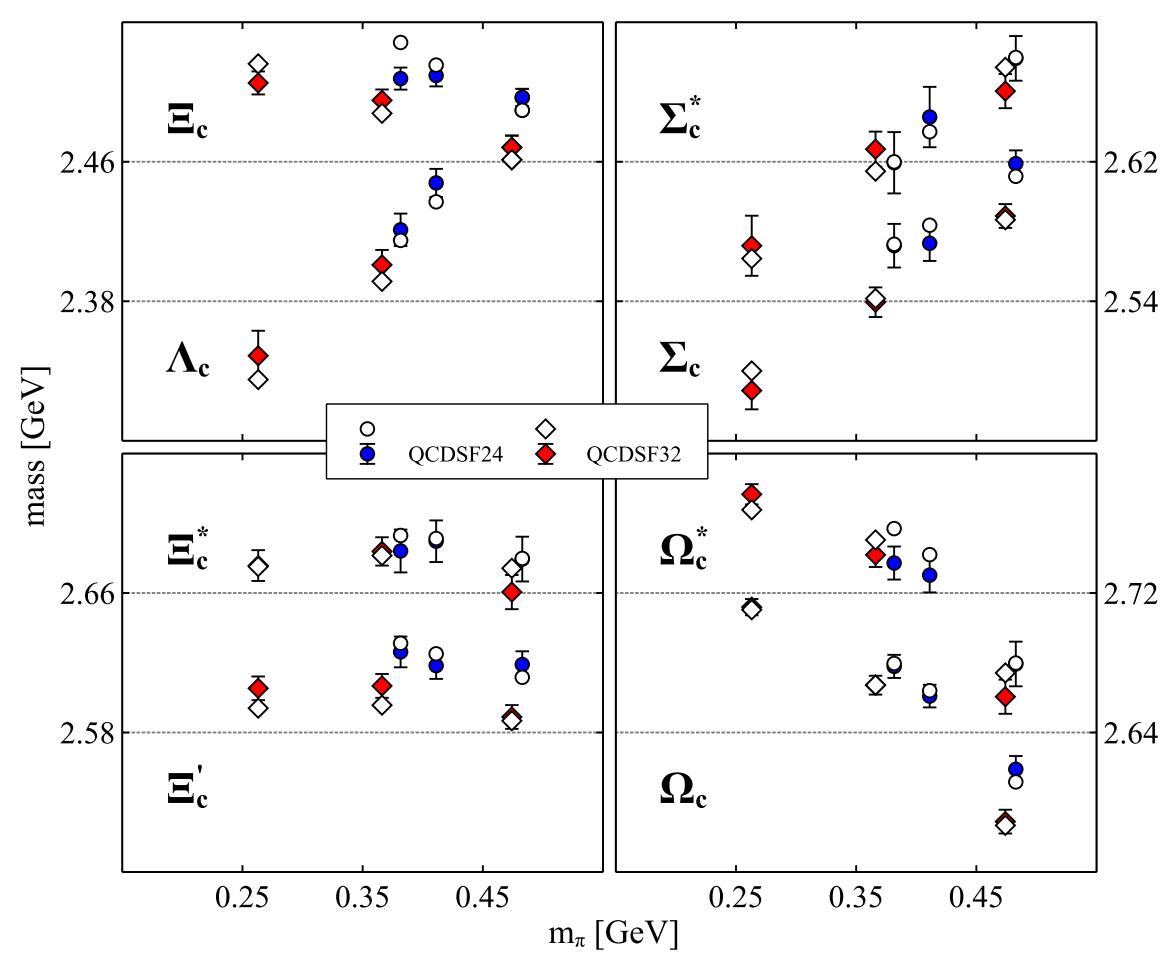

FIG. 1. Our results from Fit 1 for the charmed baryon masses on the QCDSF-UKQCD ensembles [19]. The lattice results are given by blue $\left(24^{3}\right.$ lattice $)$ and red $\left(32^{3}\right.$ lattice $)$ filled symbols, where statistical errors are shown only. They are compared to the chiral extrapolation results in open symbols, which are always displayed on top of the lattice points.

In addition, we introduce a universal parameter $\Delta_{c}$ of the form

$$
a M_{H} \rightarrow a M_{H}+\left(1+\epsilon_{H}\right) a \Delta_{c}, \quad \text { with } \quad \epsilon_{H} \simeq 0,
$$

with which the choice of the charm-quark mass can be finetuned [3]. The values of $\epsilon_{H}$ depend not only on the light quark masses and the type of charmed baryon considered, but also on the $\beta_{\mathrm{QCD}}$ value of the ensemble considered. Given the charmed baryon masses at two distinct values of the charm-quark mass, $\Delta_{c}$ and $\epsilon_{H}$ can be determined [3]. If such data are not available, we can access the parameters $\Delta_{c}$ only, i.e., we assume $\epsilon_{H}=0$ in this case. Nonvanishing values for $\Delta_{c}$ hint at a possible mismatch of the chosen charm-quark mass in a given lattice ensemble or a leadingorder discretization effect as it may be implied by our nonstandard scale setting approach.

In Tables I and II, we collect the values of the LEC according to three fit scenarios. Sets of independent LEC are shown only. The remaining ones follow from the NLO large- $N_{c}$ sum rules. Our Fit 1 is our most reasonable scenario with an excellent reproduction of the lattice datasets. It rests on a systematical error of $10 \mathrm{MeV}$ which was chosen such as to arrive at about $\chi^{2} / N \sim 1$, with $N=210$ the number of fitted baryon masses. We find $\chi^{2} /$ d.o.f. $\simeq 1.01$ and $\chi^{2} /$ d.o.f. $\simeq 2.44$ in the presence and absence of that systematical error, respectively. Similar results are observed for the Fit 2 scenario which rely on a systematical error ansatz of $5 \mathrm{MeV}$ instead. Here we have $\chi^{2} /$ d.o.f. $\simeq 1.55$ and $\chi^{2} /$ d.o.f. $\simeq 2.14$. In Fit 3 , we explore a parameter set which does not quite match the success of Fit 1 and Fit 2 in the reproduction of the lattice data with $\chi^{2} /$ d.o.f. $\simeq 1.14$ and $\chi^{2} /$ d.o.f. $\simeq 2.77$. Fit 3 is based on a negative value of $F_{[\overline{3} 6]}=-0.753$.

For all three scenarios, we find a variation of about $5 \%$ in $\chi^{2} / N$ if a subset of lattice data with $m_{\pi}<300 \mathrm{MeV}$, $m_{\pi}<400 \mathrm{MeV}$, or $m_{\pi}<500 \mathrm{MeV}$ is considered only. This is consistent with the expectation that in a flavor SU(3) framework results should not turn more accurate as we lower the pion mass only. This is so since the available lattice dataset rests on strange-quark masses around its physical value. Therefore, fits to the current lattice dataset at different chiral orders cannot be well justified at this stage. We investigated further partial $\chi^{2} / N$ values that arise if different species of the baryons are considered only. Here we find a variation of less than $20 \%$ for Fit 1 and Fit 3, but significantly larger values up to $50 \%$ for Fit 2 .

We wish to emphasize an important finding. For the LEC in Fit 1 and Fit 2, we observe that the hierarchy of the large$N_{c}$ sum rules is well followed. This is not the case for the Fit 3 scenario. For instance, at LO, we expect $F_{[\overline{3} \overline{3}]}=0$, $b_{1, \overline{3} 6]}=c_{n,[\overline{3} 6]}=0$, and $c_{n,[66]}=e_{n,[66]}$. Further such relations can be traced in [4]. Our values for the low-energy constants, $L_{i}$, in Fit 1 and Fit 2 are compatible with our previous estimates $[2,3]$, but also with the ranges from the FLAG report [25].

In Fig. 1, we confront the implications of Fit 1 on the baryon masses as computed by the QCDSF-UKQCD 


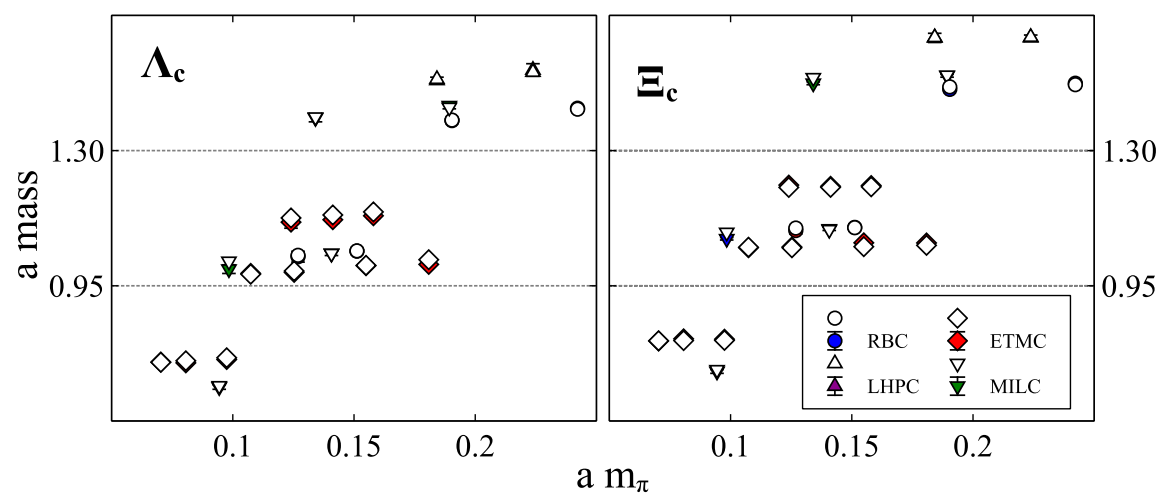

FIG. 2. Our results Fit 1 for the charmed baryon masses on the LHPC [13], RBC-UKQCD [17], MILC-HISQ [14], and ETMC [18] lattice setup. The extrapolation results in open symbols are always displayed on top of the colored lattice points.

Collaboration [19]. Further results are shown in Figs. 2 and 3 for the baryon masses based on RBC-UKQCD [17] and ETMC [18] ensembles. Additional results from [13,14] we label by LHPC and MILC in the figures. While [13] uses the MILC-Asqtad ensembles, [14] uses the MILCHISQ ensembles for the sea quarks. Both studies rely on a domain-wall formalism for the valence quarks. We use the label LHPC for [13] since it is based on the lattice setup of that collaboration. All such lattice data are well reproduced.

In Table III, we present the results for the offset parameters, $\Delta_{c}$, as introduced in (6), on ensembles of QCDSF-UKQCD, LHPC, and RBC-UKQCD as they

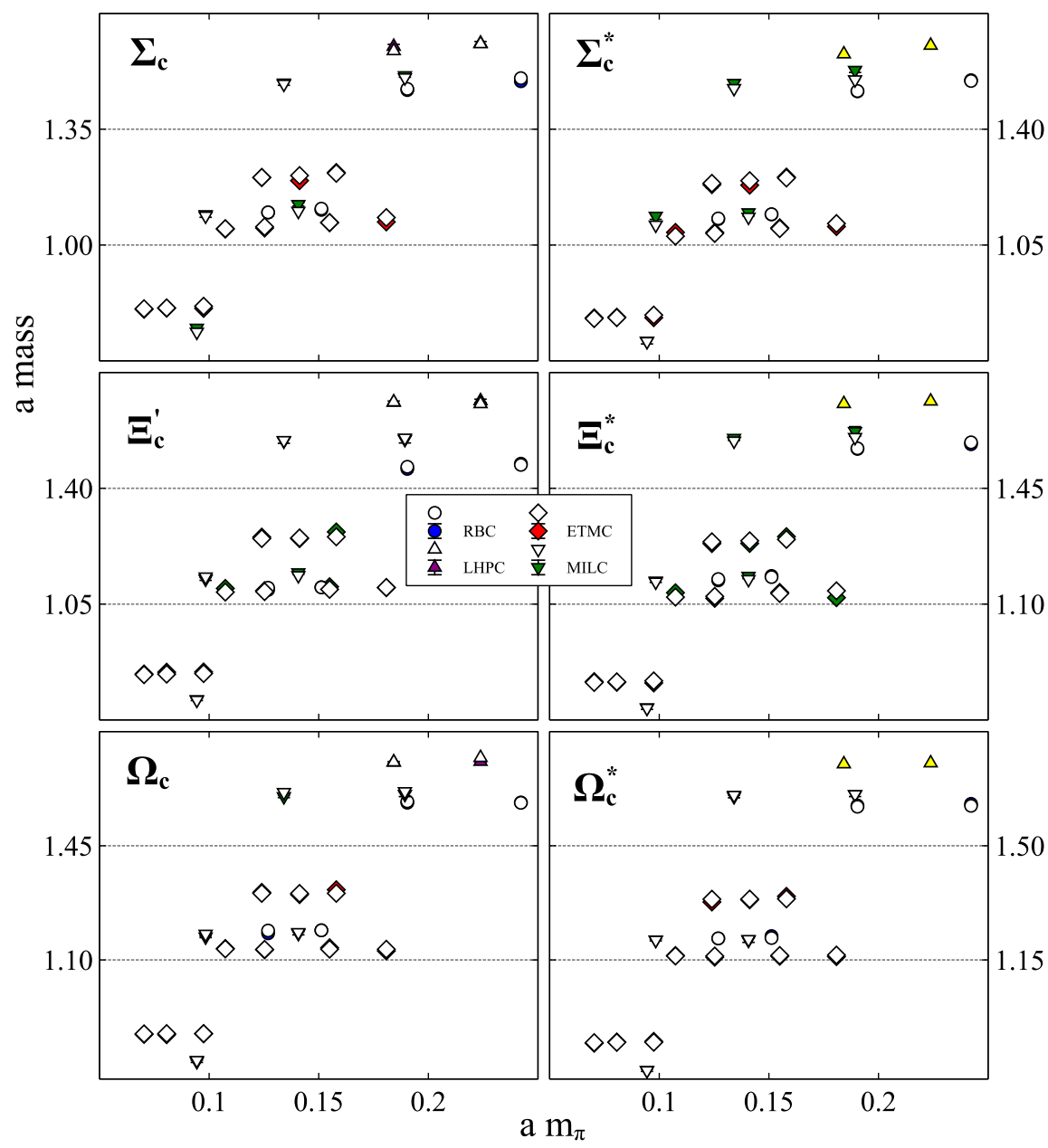

FIG. 3. Continuation of Fig. 2. 
TABLE III. Specifics for Fits 1-3. The offset parameter $a \Delta_{c}$ is introduced in (6). While Fit 1 and Fit 3 are based on a systematical error ansatz of $10 \mathrm{MeV}$, Fit 2 rests on $5 \mathrm{MeV}$.

\begin{tabular}{|c|c|c|c|c|}
\hline & Fit 1 & Fit 2 & Fit 3 & Lattice \\
\hline$a_{\mathrm{QCDSF}-\mathrm{UKQCD}}[\mathrm{fm}]$ & $0.0727\left(\begin{array}{l}+1 \\
-0\end{array}\right)$ & $0.0734\left(\begin{array}{l}+1 \\
-4\end{array}\right)$ & $0.0738\left(\begin{array}{l}+1 \\
-1\end{array}\right)$ & $0.072(4)[19]$ \\
\hline$a \Delta_{c, \mathrm{QCDSF}-\mathrm{UKQCD}}$ & $-0.0198\left(\begin{array}{c}+9 \\
-2\end{array}\right)$ & $-0.0064\left(\begin{array}{l}+09 \\
-47\end{array}\right)$ & $-0.0032\left({ }_{-4}^{+6}\right)$ & \\
\hline$\chi^{2} / N$ & 0.40 & 0.63 & 0.62 & \\
\hline$a_{\text {LHPC }}[\mathrm{fm}]$ & $0.1282\left({ }_{-1}^{+1}\right)$ & $0.1273\left({ }_{-4}^{+1}\right)$ & $0.1276\left({ }_{-4}^{+2}\right)$ & $0.1243(25)[13]$ \\
\hline$a \Delta_{c, \mathrm{LHPC}}$ & $0.0200\left(\begin{array}{c}+00 \\
-10\end{array}\right)$ & $0.0200\left({ }_{-34}^{+00}\right)$ & $-0.0200\left({ }_{-32}^{+00}\right)$ & \\
\hline$\chi^{2} / N$ & 0.28 & $0.75^{-34}$ & 0.68 & \\
\hline$a_{c, \mathrm{RBC}-\mathrm{UKQCD}}^{\beta \simeq 6.76}[\mathrm{fm}]$ & $0.1179\left({ }_{-1}^{+1}\right)$ & $0.1186\left({ }_{-2}^{+0}\right)$ & $0.1186\left({ }_{-1}^{+0}\right)$ & $0.1119(17)[17]$ \\
\hline$a \Delta_{c, \mathrm{RBC}-\mathrm{UKQCD}}^{\beta \simeq 6.76}$ & $0.0320\left({ }_{-36}^{+22}\right)$ & $0.0493\left({ }_{-83}^{+88}\right)$ & $0.0443\left({ }_{-14}^{+65}\right)$ & \\
\hline$\chi^{2} / N$ & 0.42 & 0.78 & 0.80 & \\
\hline$a_{c, \mathrm{RBC}-\mathrm{UKQCD}}^{\beta \simeq 7.09}[\mathrm{fm}]$ & $0.0885\left({ }_{-1}^{+0}\right)$ & $0.0890\left({ }_{-2}^{+1}\right)$ & $0.0888\left({ }_{-1}^{+1}\right)$ & $0.0849(17)[17]$ \\
\hline$a \Delta_{c, \mathrm{RBC}-\mathrm{UKQCD}}^{\beta \simeq 7.09}$ & $0.0267\left({ }_{-26}^{+14}\right)$ & $0.0384\left({ }_{-61}^{+52}\right)$ & $0.0335\left(\begin{array}{c}+58 \\
-09\end{array}\right)$ & \\
\hline$\chi^{2} / N$ & 0.48 & 1.00 & 0.61 & \\
\hline
\end{tabular}

follow in our fit scenarios. In addition, the lattice scales and the quality of the data reproduction are quantified. We find significant sizes for the offset parameters. Note, however, that from those results we cannot infer a mismatch of the chosen charm-quark mass. This is so since we cannot exclude the presence of discretization effects which may be parametrized by a contribution to $\Delta_{c} \sim a$ proportional to the lattice scale. A minimal model to discriminate an offset in the charm-quark mass from the presence of discretization effects is

$$
a M_{H} \rightarrow a M_{H}+a \bar{\Delta}_{c}+a^{2} \bar{\Delta}_{d}
$$

where the parameters $\bar{\Delta}_{c}$ and $\bar{\Delta}_{d}$ should not depend on the chosen ensemble for a given lattice setup. While the term

TABLE IV. Continuation of Table III.

\begin{tabular}{|c|c|c|c|c|}
\hline & Fit 1 & Fit 2 & Fit 3 & Lattice \\
\hline$a_{c, \mathrm{MILC}}^{\beta=6.00}[\mathrm{fm}]$ & $0.1234\left({ }_{-3}^{+2}\right)$ & $0.1210\left({ }_{-2}^{+4}\right)$ & $0.1230\left(\begin{array}{l}+1 \\
-1\end{array}\right)$ & $0.1193(9)[14]$ \\
\hline$a \Delta_{c, \mathrm{MILC}}^{\beta=6.00}$ & $0.0493\left({ }_{-70}^{+30}\right)$ & $0.0285\left(\begin{array}{l}+186 \\
-166\end{array}\right)$ & $0.0461\left({ }_{-8}^{+4}\right)$ & \\
\hline$\chi^{2} / N$ & 1.41 & 2.11 & 0.78 & \\
\hline$a_{\mathrm{MILC}}^{\beta=6.30}[\mathrm{fm}]$ & $0.0903\left({ }_{-4}^{+1}\right)$ & $0.0885\left({ }_{-1}^{+5}\right)$ & $0.0904\left({ }_{-0}^{+0}\right)$ & $0.0871(11)[14]$ \\
\hline$a \Delta_{c, \mathrm{MILC}}^{\beta=6.30}$ & $0.0295\left({ }_{-47}^{+17}\right)$ & $0.0147\left({ }_{-093}^{+134}\right)$ & $0.0332\left(\begin{array}{l}+3 \\
-6\end{array}\right)$ & \\
\hline$\chi^{2} / N$ & 1.96 & 2.90 & 1.50 & \\
\hline$a_{\mathrm{MILC}}^{\beta=6.72}[\mathrm{fm}]$ & $0.0603\left(\begin{array}{l}+1 \\
-3\end{array}\right)$ & $0.0592\left({ }_{-1}^{+4}\right)$ & $0.0607\left(\begin{array}{l}+0 \\
-2\end{array}\right)$ & $0.0578(23)[14]$ \\
\hline$a \Delta_{c, \mathrm{MILC}}^{\beta=6.72}$ & $0.0157\left({ }_{-29}^{+09}\right)$ & $0.0061\left({ }_{-45}^{+89}\right)$ & $0.0218\left({ }_{-3}^{+2}\right)$ & \\
\hline$\chi^{2} / N$ & 0.44 & 0.64 & 0.47 & \\
\hline$a_{c, \mathrm{ETMC}}^{\beta=1.90}[\mathrm{fm}]$ & $0.0956\left({ }_{-1}^{+1}\right)$ & $0.0959\left({ }_{-4}^{+2}\right)$ & $0.0965\left({ }_{-1}^{+1}\right)$ & $0.0936(13)[18]$ \\
\hline$a \Delta_{c, \mathrm{ETMC}}^{\beta=1.90}$ & $-0.0497\left({ }_{-11}^{+11}\right)$ & $-0.0400\left({ }_{-50}^{+11}\right)$ & $-0.0347\left(_{-09}^{+11}\right)$ & \\
\hline$\chi^{2} / N$ & 1.06 & 1.50 & 1.19 & \\
\hline$a_{\text {ETMC }}^{\beta=1.95}[\mathrm{fm}]$ & $0.0832\left(\begin{array}{l}+1 \\
-0\end{array}\right)$ & $0.0834\left({ }_{-3}^{+1}\right)$ & $0.0838\left({ }_{-0}^{+0}\right)$ & $0.0823(10)[18]$ \\
\hline $\begin{array}{l}a \Delta_{c, \mathrm{ETMC}}^{\beta=1.95} \\
\text { ETM }\end{array}$ & $-0.0406\left({ }_{-9}^{+9}\right)$ & $-0.0331\left({ }_{-43}^{+09}\right)$ & $-0.0301\left(\begin{array}{l}+9 \\
-8\end{array}\right)$ & \\
\hline$\chi^{2} / N$ & 1.18 & 1.70 & 1.46 & \\
\hline$a_{\text {ETMC }}^{\beta=2.10}[\mathrm{fm}]$ & $0.0643\left({ }_{-1}^{+1}\right)$ & $0.0644\left(\begin{array}{l}+1 \\
-2\end{array}\right)$ & $0.0646\left({ }_{-1}^{+0}\right)$ & $0.0646(7)[18]$ \\
\hline$a \Delta_{c, \mathrm{ETMC}}^{\beta=2.10}$ & $-0.0283\left({ }_{-7}^{+7}\right)$ & $-0.0236\left({ }_{-32}^{+06}\right)$ & $-0.0232\left(\begin{array}{l}+6 \\
-6\end{array}\right)$ & \\
\hline$\chi^{2} / N$ & 0.53 & 0.78 & 0.74 & \\
\hline
\end{tabular}




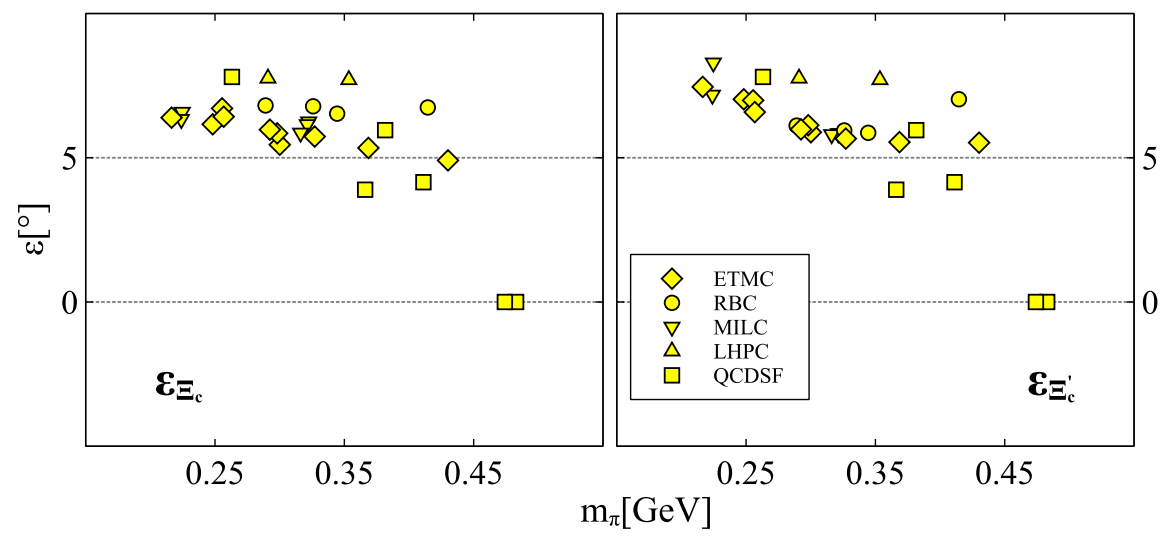

FIG. 4. The $\Xi_{c}-\Xi_{c}^{\prime}$ mixing angles on the considered lattice ensembles.

proportional to $\bar{\Delta}_{c}$ accounts for fine-tuning of the charmquark mass, the $\bar{\Delta}_{d}$ term models discretization effects. All results of our current work are based on the ansatz (7). In particular, the lattice data as shown in Figs. 2 and 3 do consider such correction terms. The values of $\bar{\Delta}_{c}$ and $\bar{\Delta}_{d}$ can be derived from the values of the lattice scale $a$ and the offset parameters $\Delta_{c}$ as given in Tables III and IV. We find $\bar{\Delta}_{c}^{\mathrm{RBC}-\mathrm{UKQCD}}=77_{-5}^{+1} \mathrm{MeV}$ and $95_{-13}^{+2} \mathrm{MeV}$ from Fit 1 and Fit 2, respectively. We turn to the ensembles of ETMC and MILC, both of which are available at three distinct beta values. In Table IV, our results for the offset parameters, lattice scales, and chisquare values are presented. From Fit 1 , we find $\bar{\Delta}_{c}^{\mathrm{MILC}}=25_{-8}^{+1} \mathrm{MeV}$ and $\bar{\Delta}_{c}^{\mathrm{ETMC}}=-55_{-2}^{+2} \mathrm{MeV}$. Corresponding values from Fit 2 are $-5_{-4}^{+29}$ and $-52_{-9}^{+1} \mathrm{MeV}$.

In Fig. 4, we show the $\Xi_{c}-\Xi_{c}^{\prime}$ mixing angles evaluated on all considered lattice ensembles in Fit 1. Given our approach, the mixing angle acquires a mass dependence, and therefore two distinct values are implied. At the physical point, the mixing angles reach $\epsilon_{\Xi} \simeq 0.103\left({ }_{-4}^{+3}\right)$ and $\epsilon_{\Xi_{c}^{\prime}} \simeq 0.117\left({ }_{-2}^{+2}\right)$ at the $\Xi_{c}$ and $\Xi_{c}^{\prime}$ masses, respectively. We find rather small values for the mixing angles on all considered lattice ensembles.

We wish to comment on the uncertainties in the LEC of Tables I-II. We derived the one-sigma statistical errors. Corresponding errors in Tables III and IV are derived for the various lattice scales and offset parameters. Since a quite large set of data points is fitted in terms of a significantly smaller number of LEC, any derived statistical error is of almost no physical relevance. Uncertainties are largely dominated by the systematic uncertainties, which can be made quantitative only after a better control of discretization effects and the generic form of two-loop contributions are available.

To conclude, the size of the errors in the LEC as obtained in our study is indicative only since it reflects an ad hoc assumption on the form of the systematic error. Further lattice data and detailed studies are required. Once lattice data are available at small pion and kaon masses, the convergence properties of the chiral flavor SU(3) expansion can be scrutinized in significant depth.

\section{SUMMARY AND OUTLOOK}

In this work, we documented a first chiral extrapolation fit to the world data on charmed baryon masses on QCD lattices. The set of low-energy constants that determine the baryon masses at $\mathrm{N}^{3} \mathrm{LO}$ was obtained. Given the latter, the way is paved to explore the coupled-channel systems of Goldstone bosons and charmed baryon ground states. Such systems are of particular importance in QCD since, like in the open-charm meson sector, flavor exotic multiplets are expected here.

\section{ACKNOWLEDGMENTS}

Constantia Alexandrou, Gunnar Bali, John Bulava, Utku Can, and Daniel Mohler are acknowledged for stimulating discussions. Y. H. received partial support from Suranaree University of Technology, Office of the Higher Education Commission under NRU project of Thailand (SUT-COE: High Energy Physics and Astrophysics) and SUT-CHENRU (Grant No. FtR.11/2561). 
[1] R. A. Briceno, J. J. Dudek, and R. D. Young, Rev. Mod. Phys. 90, 025001 (2018).

[2] M. F. M. Lutz, Y. Heo, and X.-Y. Guo, Nucl. Phys. A977, 146 (2018).

[3] X.-Y. Guo, Y. Heo, and M. F. M. Lutz, Phys. Rev. D 98, 014510 (2018).

[4] Y. Heo, X.-Y. Guo, and M. F. M. Lutz, Phys. Rev. D 98, 054012 (2018).

[5] X.-Y. Guo and M. F. M. Lutz, Nucl. Phys. A988, 48 (2019).

[6] X.-Y. Guo and M. F. M. Lutz, Nucl. Phys. A988, 36 (2019).

[7] T.-M. Yan, H.-Y. Cheng, C.-Y. Cheung, G.-L. Lin, Y. C. Lin, and H.-L. Yu, Phys. Rev. D 46, 1148 (1992).

[8] P. L. Cho, Phys. Lett. B 285, 145 (1992).

[9] M. F. M. Lutz and E. E. Kolomeitsev, Nucl. Phys. A730, 110 (2004).

[10] M. F. M. Lutz, D. Samart, and Y. Yan, Phys. Rev. D 90, 056006 (2014).

[11] J. Hofmann and M. F. M. Lutz, Nucl. Phys. A763, 90 (2005).

[12] J. Hofmann and M. F. M. Lutz, Nucl. Phys. A776, 17 (2006).

[13] L. Liu, H.-W. Lin, K. Orginos, and A. Walker-Loud, Phys. Rev. D 81, 094505 (2010).

[14] R. A. Briceno, H.-W. Lin, and D. R. Bolton, Phys. Rev. D 86, 094504 (2012).
[15] C. Alexandrou, J. Carbonell, D. Christaras, V. Drach, M. Gravina, and M. Papinutto, Phys. Rev. D 86, 114501 (2012).

[16] Y. Namekawa et al. (PACS-CS Collaboration), Phys. Rev. D 87, 094512 (2013).

[17] Z. S. Brown, W. Detmold, S. Meinel, and K. Orginos, Phys. Rev. D 90, 094507 (2014).

[18] C. Alexandrou, V. Drach, K. Jansen, C. Kallidonis, and G. Koutsou, Phys. Rev. D 90, 074501 (2014).

[19] P. Perez-Rubio, S. Collins, and G. S. Bali, Phys. Rev. D 92 , 034504 (2015).

[20] Y. Heo and M. F. M. Lutz, Phys. Rev. D 97, 094004 (2018).

[21] E. Jenkins, Phys. Rev. D 54, 4515 (1996).

[22] M. F. M. Lutz, R. Bavontaweepanya, C. Kobdaj, and K. Schwarz, Phys. Rev. D 90, 054505 (2014).

[23] R. Berlich, S. Gabriel, A. Garcia, and M. Kunze, Data Driven e-Science, Conference proceedings of ISGC 2010 (Springer, New York, 2010), p. 303.

[24] C. Patrignani et al. (Particle Data Group), Chin. Phys. C 40, 100001 (2016).

[25] S. Aoki et al. (Flavour Lattice Averaging Group), Eur. Phys. J. C 80, 113 (2020).

[26] C. Alexandrou, K. Hadjiyiannakou, and C. Kallidonis, Phys. Rev. D 94, 034502 (2016). 